Total Synthesis

\title{
A Cascade Strategy Enables a Total Synthesis of (-)-Gephyrotoxin**
}

\author{
Shuyu Chu, Stephen Wallace and Martin D. Smith*
}

\begin{abstract}
A concise and efficient synthesis of (-)-gephyrotoxin from (L)-pyroglutaminol has been realized. The key step in this approach is a diastereoselective intramolecular enamine-Michael cascade that forms two rings and two stereocenters and generates a stable tricyclic iminium cation. A hydroxyl-directed reduction of this intermediate plays a key role in establishing the required cisdecahydroquinoline ring system, enabling the total synthesis of (-)gephyrotoxin in 9 steps and 14\% overall yield. The absolute configuration of our synthetic material was confirmed by single crystal X-ray diffraction and is consistent with the structure originally proposed for naturally isolated material.
\end{abstract}

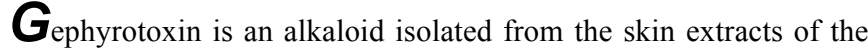
Columbian poison dart frog Dendrobates histrionicus, and possesses an interesting perhydropyrroloquinoline core and five stereocentres. ${ }^{[1]}$ Unlike many other dendrobatid alkaloids, gephyroxin is relatively non-toxic ${ }^{[2]}$ but does exhibit complex effects on transmission at the neuromuscular junction ${ }^{[3,4]}$ and weak antimuscarinic actions, despite not interacting with the acetylcholine binding site. ${ }^{[5,6]}$ The low natural abundance and unique neurological profile of gephyrotoxin has led to significant synthetic interest in these alkaloids, culminating in four elegant and inventive total syntheses from the laboratories of Kishi, ${ }^{[7]}$ Hart, ${ }^{[8]}$ Overman, ${ }^{[9]}$ and Sato and Chida, ${ }^{[10]}$ and numerous formal syntheses that intersect with an intermediate from Kishi's approach. ${ }^{[11]}$ We rationalized that the tricyclic core of gephyrotoxin could be constructed from a cisdisubstituted pyrrolidine fragment bearing an alcohol side chain (scheme 1). Elaboration of this fragment to incorporate a ketone (at $\mathrm{C}-10$ ) and an enone (at $\mathrm{C}-14$ ) could, upon liberation of a nucleophilic pyrrolidine amine, permit a cascade cyclization that could generate the entire tricyclic framework in a single operation. ${ }^{[12]}$ In this scenario, the $\mathrm{C}-2$ alcohol plays a key stereodirecting role for reduction of a $\mathrm{C}-10$ iminium cation to afford the cis-decahydroquinoline. Strategically, cascade reactions are

[*] Shuyu Chu, Dr Stephen Wallace, Prof. Dr Martin D. Smith

Chemistry Research Laboratory

University of Oxford

12 Mansfield Road, Oxford, OX1 3TA (UK)

E-mail: martin.smith@chem.ox.ac.uk

Homepage: http://msmith.chem.ox.ac.uk

[**] The European Research Council has provided financial support (FP7/2007-2013) / ERC grant agreement no. 259056. We gratefully acknowledge the Diamond Light Source for an award of instrument time on 119 (MT9981) and thank John Jolliffe for help with X-ray crystallography. We are extremely grateful to Professor Yoshito Kishi (Harvard University) for helpful discussions and exchange of information.

Supporting information for this article is available on the WWW under http://dx.doi.org/10.1002/anie.201xxxxxx.

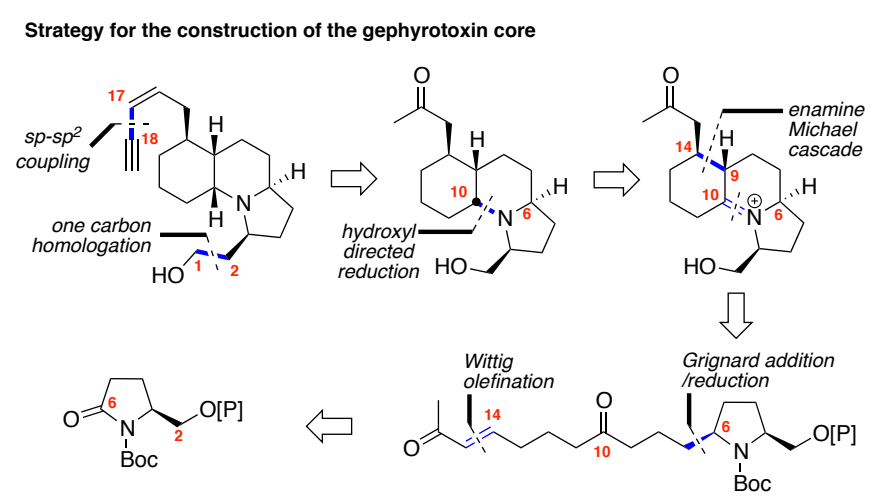

Scheme 1. Strategy for the synthesis of (-)-gephyrotoxin.

attractive as they offer the potential for the rapid generation of molecular complexity, which can shorten routes to natural productlike materials. ${ }^{[13]}$ However, this must be balanced with the need for chemo- and stereoselectivity, and redox economy in order to not compromise overall efficiency. ${ }^{[14]}$

The route begins with $N$ - and $O$-protection of Lpyroglutaminol $\mathbf{1}$, which could be performed in a one-pot sequence in $84 \%$ overall yield to afford 2 (scheme 2 ). ${ }^{[15]}$ The integrity of the C-3 stereocentre in $\mathbf{2}$ was established by chiral stationary phase HPLC ( $>99: 1$ e.r.), and optical rotation confirmed absolute configuration. ${ }^{[16]}$ Treatment of $\mathbf{2}$ with a cyclopentene-containing Grignard reagent, conceived as a masked C-10/C-14 dicarbonyl unit, afforded an inconsequential mixture of C-6 diastereoisomers, which was reduced in situ with L-selectride in the presence of $\mathrm{BF}_{3} \cdot \mathrm{OEt}_{2} \cdot{ }^{[17]}$ The 3,6-cis-pyrrolidine $\mathbf{3}$ was generated exclusively, presumably via hydride delivery to the least hindered face of the in situ generated $\mathrm{N}$ acyliminium cation. The C-10/C-14 dicarbonyl unit was unmasked with ozone, and the resultant terminal aldehyde $\mathbf{4}$ homologated in situ to a trans-configured $\alpha, \beta$-unsaturated ketone 5 using 1(triphenylphosphoranylidene)-2-propanone. This afforded the key cascade precursor for the generation of the gephyrotoxin core. Removal of the tert-butoxycarbonyl group was cleanly achieved with TFA in DCM at room temperature; this also led to hydrolysis of the primary tert-butyldimethylsilyl ether. The excess TFA was removed and subsequent warming of a chloroform solution to $40^{\circ} \mathrm{C}$ for $72 \mathrm{~h}$ led to the formation of a tricyclic iminium cation 7 , exclusively with the required C-9 and C-14 configuration. This transformation likely proceeds via intramolecular condensation of the pyrrolidine amine onto the $\mathrm{C}-10$ ketone to afford a bicyclic enamine 6 that undergoes a diastereoselective intramolecular Michael addition onto the C-14 enone. ${ }^{[18]}$ The stereochemistry of this transformation can be rationalized through a chair-like transition state in which the Michael acceptor adopts a pseudoequatorial orientation to minimize diaxial interactions, with facial discrimination of the bicyclic enamine a consequence of the hydroxymethyl substituent and its effect on the conformation of the 


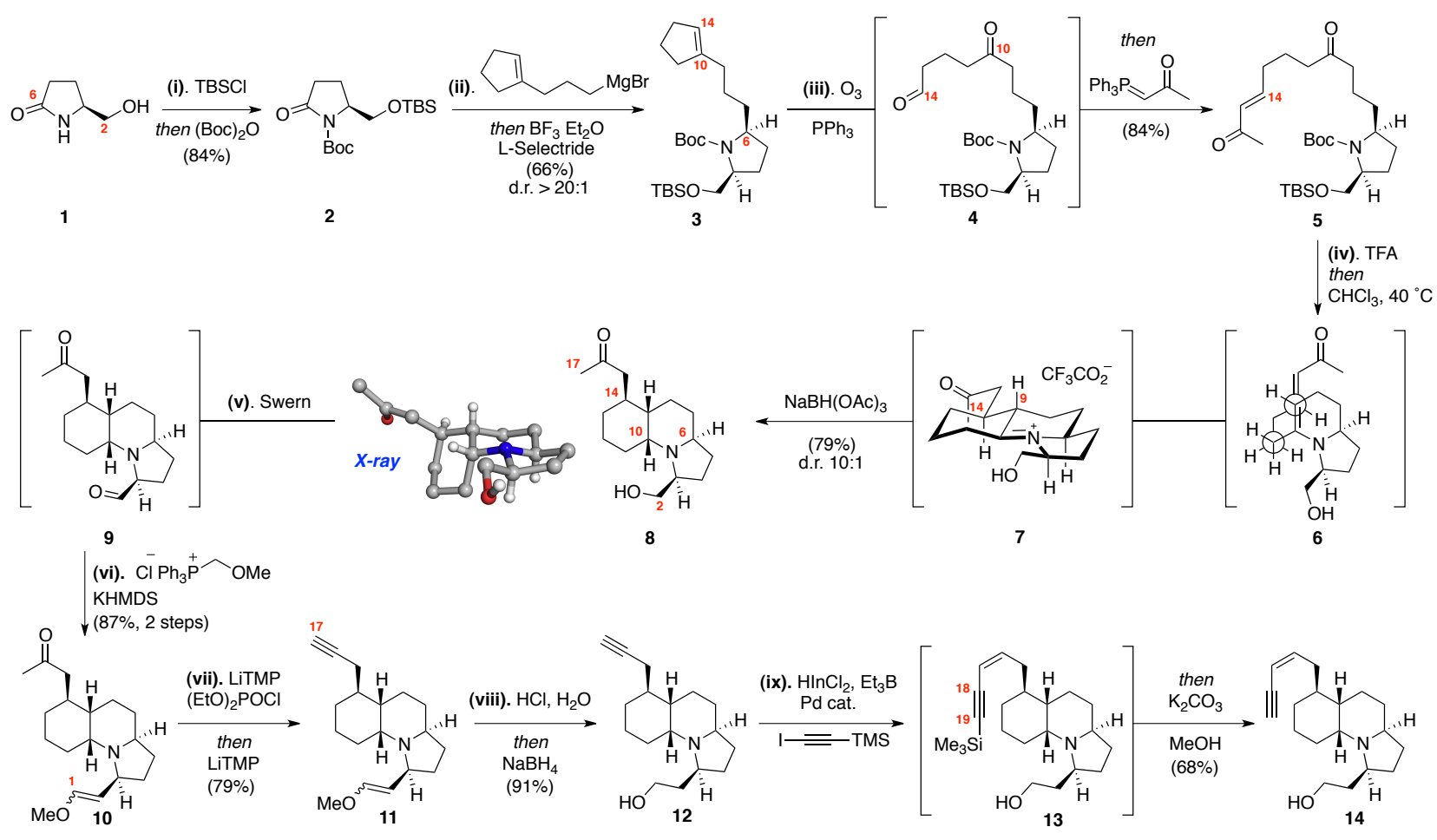

Scheme 2. Total synthesis of gephyrotoxin: (i). TBSCl, (1.15 eq.), KHMDS (1.2 eq.), MeCN, RT; then Boc $\mathrm{O}_{2}$ (3.0 eq.), DMAP (0.05 eq.), RT. (ii). Grignard (2.0 eq.), $\mathrm{THF},-78^{\circ} \mathrm{C} ; \mathrm{BF}_{3} . \mathrm{Et}_{2} \mathrm{O}$ (6.0 eq.), L-Selectride (2.0 eq.), $-78^{\circ} \mathrm{C}$ to $\mathrm{RT}$. (iii). $\mathrm{O}_{3}, \mathrm{DCM},-78^{\circ} \mathrm{C}$; then $\mathrm{PPh}_{3}\left(1.05\right.$ eq.), $-78^{\circ} \mathrm{C}$ to $\mathrm{RT}$; then $\mathrm{Ph}_{3} \mathrm{PCH}{ }_{2} \mathrm{COCH}{ }_{3}(1.1$ eq.), $\mathrm{RT}$. (iv). TFA/DCM (1:3, v/v), $0^{\circ} \mathrm{C}$ to $\mathrm{RT}$; then $\mathrm{CHCl}_{3}, 40^{\circ} \mathrm{C}, 72 \mathrm{~h}$; then $\mathrm{NaBH}(\mathrm{OAc})_{3}$ (1.5 eq.), DCM, RT. (v). oxalyl chloride (1.1 eq.), DMSO (2.5 eq.), Et $\mathrm{N}_{3}$ (5.0 eq.), $\mathrm{DCM},-78^{\circ} \mathrm{C}$ to RT. (vi). $\mathrm{Cl}^{-} \mathrm{Ph}_{3} \mathrm{P}^{+} \mathrm{CH}_{2} \mathrm{OMe}$ (1.2 eq.), $\mathrm{KHMDS}$ (1.15 eq.), THF, $-78^{\circ} \mathrm{C}$ to RT. (vii). LiTMP (1.05 eq.), (OEt) $\mathrm{POCl}\left(1.1 \mathrm{eq}\right.$.), THF, $-78^{\circ} \mathrm{C}$ to RT; then LiTMP (2.25 eq.), THF, $-78^{\circ} \mathrm{C}$ to RT. (viii). $\mathrm{HCl}\left(20\right.$ eq.), THF/ $\mathrm{H}_{2} \mathrm{O}, 25^{\circ} \mathrm{C}$; then $\mathrm{NaBH}_{4}\left(2.0\right.$ eq.), MeOH. (ix). $\mathrm{HInCl} 2$ (1.5 eq.), Et $\mathrm{B}_{3} \mathrm{~B}$ (1.0 eq.), AcOH (1.0 eq.), THF, $-78^{\circ} \mathrm{C}$; then TMS-iodoacetylene (1.5 eq.), $\mathrm{Pd}_{2}(\mathrm{dba})_{3} \cdot \mathrm{CHCl}_{3}\left(0.005\right.$ eq.), tri(2-furyl)phosphine $\left(0.045\right.$ eq.), $\mathrm{DMF} / \mathrm{THF}, \mathrm{reflux} ;$ then $\mathrm{K}_{2} \mathrm{CO}_{3}(50$ eq.), MeOH, $\mathrm{RT}$.

pyrrolidine ring. This tricyclic iminium species was sufficiently stable to be isolable (as its trifluoroacetate salt, d.r. $>20: 1$ ) but in practice was reduced in situ. Reduction with non-chelating hydride sources such as sodium cyanoborohydride was efficient and diastereoselective but unfortunately produced the 9,10-trans- ring junction by virtue of the stereoelectronically preferred axial delivery mode. ${ }^{[19]}$ Consequently, we explored intramolecular delivery of hydride via the tethered hydroxymethyl group at C-2 using sodium triacetoxyborohydride; ${ }^{[20]}$ a related approach has been elegantly applied by Ciufolini in the synthesis of Cylindricine C. ${ }^{[21]}$ This afforded the tricyclic gephyrotoxin core 8 with the requisite 9,10cis- stereochemistry with good diastereoselectivity (10:1, cis:trans, separable by chromatography) and in $79 \%$ overall yield from $\mathbf{5}$. The stereochemical outcome of this cascade was probed by single crystal $\mathrm{X}$-ray diffraction, which confirmed that all five stereocentres for the natural product had been installed correctly. ${ }^{[22]}$ Conversion of this intermediate to the natural product required installation of the eneyne sidechain from the $\mathrm{C}-16$ ketone and a one-carbon homologation of the C-2 alcohol. This was achieved by oxidation of the C-2 alcohol to an aldehyde under Swern conditions to afford $\mathbf{9} ;{ }^{[23]}$ in our hands this method was superior to Dess-Martin periodinane ${ }^{[24]}$ and TPAP. ${ }^{[25]}$ The aldehyde could be purified, but in practice was used crude in subsequent transformations after work-up. Treatment of aldehyde 9 with (methoxymethyl)triphenylphosphonium chloride and KHMDS in THF afforded vinyl ether $\mathbf{1 0}$ as an inconsequential 1:1 mixture of geometrical isomers. The ketone functional group in 10 was transformed into an intermediate enol phosphate through kinetic deprotonation with lithium tetramethyl piperidine in THF and trapping with diethylphosphoryl chloride; subsequent addition of excess lithium tetramethylpiperidine led to elimination to afford terminal alkyne 11, consistent with the method described by Negishi. ${ }^{[26]}$ Hydrolysis of the methyl enol ether was achieved by stirring in aqueous $\mathrm{HCl}$, affording a $\mathrm{C}-1$ aldehyde which, after removal of aqueous THF and addition of methanol, was reduced with sodium borohydride to afford $\mathrm{C}-1$ alcohol $\mathbf{1 2}$. The final carboncarbon bond in the synthesis was formed through a hydrometalation/cross-coupling strategy. Trans-hydrometallation of $\mathbf{1 2}$ by in situ generated dichloroindium hydride in the presence of triethylborane generated a vinyl indium species; this was crosscoupled with trimethylsilyl-protected iodoacetylene in the presence of pre-mixed tris(dibenzylideneacetone)dipalladium $(0)$ and tri(2furyl)phosphine to afford 13. ${ }^{[27]}$ Work-up with potassium carbonate and methanol effected terminal desilylation to afford (-)gephyrotoxin in $68 \%$ yield from $\mathbf{1 2}$. Synthetic gephyrotoxin $\mathbf{1 4}$ exhibited identical ${ }^{1} \mathrm{H}$ and ${ }^{13} \mathrm{C}$ NMR spectroscopic data to that published for authentic naturally obtained material. ${ }^{[28]}$ Based on the enantiopure L-pyroglutaminol starting material, we were confident that the absolute configuration of our synthetic gephyrotoxin was as depicted by structure $14 .{ }^{[29,30]}$ To further investigate this, we crystallized the hydrochloride salt of our synthetic 14, which confirmed its relative and absolute configuration (Figure 1). Measurement of the optical rotation of synthetic 14 gave $[\alpha]_{20}^{D}=$ $-52.3(\mathrm{c}=1.0, \mathrm{EtOH}) .{ }^{[31]}$ This is consistent with the value reported for material isolated from natural sources $\left([\alpha]_{20}^{D}=-51.5(\mathrm{c}=1.0\right.$, EtOH)) in which absolute configuration was assigned though X-ray analysis of a single crystal of gephyrotoxin hydrobromide. However, this value is opposite in sign to that reported by Kishi for synthetic material of the same proposed absolute configuration 
$\left([\alpha]_{20}^{D}=+50.0(\mathrm{c}=1.0, \mathrm{EtOH})\right) \cdot{ }^{[32]}$ On this basis, it was suggested that the absolute configuration of the natural product should be revised. ${ }^{[7 b, 33,34]}$ Unfortunately, a sample of natural gephyrotoxin is not available for direct comparison with our synthetic material and hence we cannot unequivocally confirm the absolute configuration of the natural product.

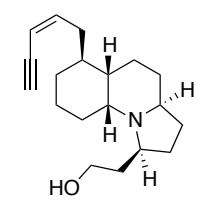

(-)-Gephyrotoxin 14 Synthetic Material: $[\alpha]_{20}^{\mathrm{D}}-52.4(\mathrm{c}=1.0, \mathrm{EtOH})$

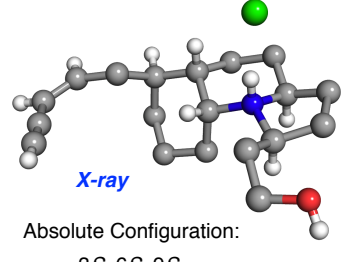

$3 S, 6 S, 9 S$, $10 R, 14 S$
Figure 1. Confirmation of absolute configuration: X-ray structure of $\mathrm{HCl}$ salt of synthetic material 14 (some hydrogen atoms omitted for clarity).

A synthesis of (-)-gephyrotoxin $\mathbf{1 4}$ has been achieved in 9 steps and $14 \%$ overall yield from L-pyroglutaminol 1. Key features of the synthesis are the diastereoselective intramolecular enamineMichael cascade (which generates two rings and two stereocentres in a single operation) and the hydroxyl-directed reduction to install the correct configuration at $\mathrm{C}-10$. From a strategic perspective, the requirement for a one-carbon homologation of the primary alcohol (from 8) is both redox and step inefficient, and represents a limitation in current methods. ${ }^{[35,36]}$ However, this requirement does permit reagent directed cis- or trans-selective reduction of iminium species 7 to allow the synthesis of analogues. Consequently, this approach compares well with other syntheses of gephyrotoxin, offers rapid and stereoselective access to this class of natural products and may provide a more general approach to decahydroquinoline-containing alkaloids.

Received: ((will be filled in by the editorial staff))

Published online on ((will be filled in by the editorial staff))

Keywords: gephyrotoxin $\cdot$ cascade $\cdot$ total synthesis $\cdot$ asymmetric $\bullet$ alkaloid

[1] J. W. Daly, B. Witkop, T. Tokuyama, T. Nishikawa, I. L. Karle, Helv. Chim. Acta 1977, 60, 1128.

[2] J. W. Daly, Fortschr. Chem. Org. Naturs. 1982, 41, 205.

[3] C. Souccar, W. A. Varanda, R. S. Aronstam, J. W. Daly, E. X. Albuquerque, Mol. Pharmacol. 1984, 25, 395.

[4] C. Souccar, W. A. Varanda, J. W. Daly, E. X. Albuquerque, Mol. Pharmacol. 1984, 25, 384.

[5] J. W. Daly, G. B. Brown, M. Mensah-Dwumah, C. W. Meyers, Toxicon 1978, 16, 189 .

[6] R. S Aronstam, J. W. Daly, T. F. Spande, T. K. Narayanan, E. X. Albuquerque, Neurochem Res. 1986, 11, 1227.

[7] a) R. Fujimoto, Y. Kishi, J. F. Blount, J. Am. Chem. Soc. 1980, 102, 7154; b) R. Fujimoto, Y. Kishi, Tetrahedron Lett. 1981, 22, 4197.

[8] a) D. J. Hart, J. Org. Chem. 1981, 46, 3576-3578; b) D. J. Hart, K. Kanai, J. Am. Chem. Soc. 1983, 105, 1255.

[9] a) L. E. Overman, C. Fukaya, J. Am. Chem. Soc. 1980, 102, 1454; b) L. E. Overman, D. Lesuisse, M. Hashimoto, J. Am. Chem. Soc. 1983, 105 , 5373 .

[10] K. Shirokane, T. Wada, M. Yoritate, R. Minamikawa, N. Takayama, T. Sato, N. Chida, Angew. Chem. Int. Ed. 2014, 53, 512.

[11] For formal syntheses which intersect Kishi's route, see: a) Y. Ito, E. Nakajo, M. Nakatsuka, T. Saegusa, Tetrahedron Lett. 1983, 24, 2881; b) W. H. Pearson, W.-K. Fang, J. Org. Chem. 2000, 65, 6838; c) S. Pichette, D. K. Winter, J. Lessard, C. Spino, J. Org. Chem. 2013, 78, 12532; d) L.-L. Wei, R. P. Hsung, H. M. Sklenicka, A. I. Gerasyuto, Angew. Chem. Int. Ed. 2001, 40, 1516; e) M. Santarem, C. VanucciBacqué, Gérard Lhommet, J. Org. Chem. 2008, 73, 6466; f) L. Miao, H. Shu, A. R. Noble, S. P. Fournet, E. D. Stevens, M. L. Trudell Arkivoc 2010, (Iv), 6; c); d) (e); f)

[12] A related approach to Lycopodium alkaloids was disclosed during the preparation of this manuscript: M. Azuma, T. Yoshikawa, N. Kogure, M. Kitajima, H. Takayama, J. Am. Chem. Soc., 2014, 136, 11618.

[13] For recent reviews on the application of cascade reactions in total synthesis see: a) K. C. Nicolaou, D. J. Edmonds, P. G. Bulger, Angew. Chem. Int. Ed. 2006, 45, 7134; b) K. C. Nicolaou, J. S. Chen, Chem. Soc. Rev. 2009, 38, 2993; c) E. A. Anderson, Org. Biomol. Chem. 2011, 9, 3997.

[14] T. Gaich, P. S. Baran, J. Org. Chem. 2010, 75, 4657.

[15] The enantiomer of $\mathbf{2}$ is commercially available from Sigma-Aldrich.

[16] For optical rotation details for 2 see: J. Ackermann, M. Matthes, C. Tamm, Helv. Chim. Acta 1990, 73, 122.

[17] J. B. Brenneman, R. Machauer, S. F. Martin, Tetrahedron 2004, 60, 7301.

[18] T. C. Sherwood, A. H. Trotta, S. A. Snyder, J. Am. Chem. Soc. 2014 136, 9743.

[19] R. V. Stevens, Acc. Chem. Res. 1984, 17, 289.

[20] D. A. Evans, J. S. Clark, R. Metternich, V. J. Novack, G. S. Sheppard, J. Am. Chem. Soc. 1990, 112, 866.

[21] S. Canesi, D. Bouchu, M. A. Ciufolini, Angew. Chem. Int. Ed. 2004 $43,4336$.

[22] Low temperature single X-ray diffraction data were collected for $\mathbf{8}$ and 14.HCl using beamline $\mathrm{I} 19(\mathrm{EH} 1)$ at Diamond Light Source $[\mathrm{H}$. Nowell, S. A. Barnett, K. E. Christensen, S. J. Teat, D. R. Allan, J. Synch. Rad. 2012, 19, 435]. Full crystallographic data for 8 and 14. $\mathrm{HCl}$ (in CIF format) is available as supplementary information and has been deposited with the Cambridge Crystallographic Data Centre (CCDC 1023956 and 1023957 respectively).

[23] K. Omura, D. Swern, Tetrahedron 1978, 34, 1651.

[24] D. B. Dess, J. C. Martin, J. Org. Chem. 1983, 48, 4155.

[25] S. V Ley, J. Norman, W. P. Griffith, Synthesis 1994, 639.

[26] a) E. Negishi, A. O. King, W. L. Klima, J. Org. Chem. 1980, 45, 2526; b) E. Negishi, A. O. King, J. M. Tour, Organic Syntheses 1986, 64,44 .

[27] K. Takami, H. Yorimitsu, K. Oshima, Org. Lett. 2002, 4, 2993.

[28] M. W. Edwards, A. Bax, J. Am. Chem. Soc. 1986, 108, 918.

[29] We have derivatized our synthetic (-)-gephyrotoxin 14 by $\mathrm{C}-1 O$ acylation with both enantiomers of methoxy(trifluoromethyl) phenylacetic acid. In both cases a single diastereoisomeric product was observed by ${ }^{1} \mathrm{H}$, and ${ }^{19} \mathrm{~F}$ NMR spectroscopy. These diastereoisomeric esters are non-coincident by ${ }^{1} \mathrm{H}$ and ${ }^{19} \mathrm{~F}$ NMR spectroscopy (see supplementary information for full details). This is consistent with our synthetic (-)-gephyrotoxin 14 being produced as a single enantiomer.

[30] J. A. Dale, D. L. Dull, H. S. Mosher, J. Org. Chem. 1968, 34, 2543.

[31] This is an average of three measurements.

[32] We have derivatized $\mathbf{1 2}$ to intersect with Kishi's route. Our material has the opposite sign of optical rotation to that prepared by Kishi; see supplementary information for full details.

[33] J. W. Daly, H. M. Garraffo and T. F. Spande in Alkaloids: Chemical and Biological Perspectives, Vol. 13 (Ed.: S. W. Pelletier), Pergamon, Oxford, 1999, p. 78.

[34] An intermediate from the Kishi synthesis has been successfully resynthesized in both enantiomeric forms by several groups (see references 11(a)-(f) for details). This confirms the relative and absolute configuration of this intermediate.

[35] N. Z. Burns, P. S. Baran, R. W. Hoffmann, Angew. Chem. Int. Ed. 2009, 48, 2854

[36] Installation of the correct C-1 sidechain earlier in the synthesis was achieved, but attempts to perform a directed iminium reduction on this material led to exclusive generation of the non-natural 9,10-trans stereochemistry and so this approach was abandoned. 


\section{Angewandte Communications}

\section{Total Synthesis}

Shuyu Chu, Stephen Wallace and Martin D. Smith*

Page - Page

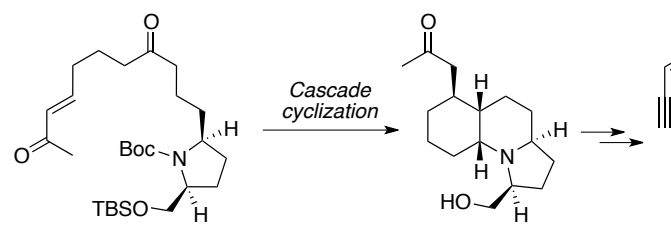

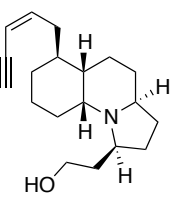

(+)-Gephyrotoxin

A Cascade Strategy Enables a Total Synthesis of (-)-Gephyrotoxin

A concise and efficient synthesis of (-)-gephyrotoxin from L-pyroglutaminol has been realized. The key step in this approach is a diastereoselective intramolecular enamime-Michael cascade that forms two rings and two stereocenters and generates a stable tricyclic iminium cation. A hydroxyl-directed reduction of this intermediate plays a key role in establishing the required cis-decahydroquinoline ring system, enabling the total synthesis of (-)-gephyrotoxin in 9 steps and $14 \%$ overall yield. 